\title{
Ecodriving for Reduction of Bus Transit Emission with Vehicle's Hybrid Dynamic Model
}

\author{
Xiuzheng Zheng ${ }^{1,2}$ and Liguo Zhang ${ }^{1,2}$ \\ ${ }^{1}$ School of Electronic and Control Engineering, Beijing University of Technology, Beijing 100124, China \\ ${ }^{2}$ Key Laboratory of Computational Intelligence and Intelligent Systems, Beijing 100124, China \\ Correspondence should be addressed to Xiuzheng Zheng; xiuzhengzheng@emails.bjut.edu.cn
}

Received 6 September 2015; Revised 12 October 2015; Accepted 15 October 2015

Academic Editor: Weiguo Xia

Copyright (c) $2015 \mathrm{X}$. Zheng and L. Zhang. This is an open access article distributed under the Creative Commons Attribution License, which permits unrestricted use, distribution, and reproduction in any medium, provided the original work is properly cited.

\begin{abstract}
This paper formulates a global ecodriving optimal control to advise the green driving speed for bus transit to minimize the exhaust emission using Vehicle-to-Infrastructure (V2I) communication. Assuming communication between vehicles and infrastructure (V2I) and knowledge of traffic signal timings and waiting passengers at stations are known, an optimal driving speed is proposed to minimize the total vehicle emissions of the bus route. The dwell time of the bus transit at each station which includes two parts is proposed. A traffic lights timing model is employed as constraints to control the formation of the green wave band. Vehicle specific power (VSP) model is further applied to evaluate the exhaust emission level linked with the speed and acceleration of the bus transit. An approximate sixteen-kilometer traffic network including fourteen intersections and fifteen stations of Beijing bus transit line 1 in Chaoyang District, Beijing, is chosen to investigate the performance of the developed ecodriving approach.
\end{abstract}

\section{Introduction}

Traffic emissions are among the biggest sources of environmental pollution in cities. The emissions of vehicles contain several harmful substances, such as nitrogen oxides $\left(\mathrm{NO}_{x}\right.$, i.e., nitrogen monoxide and nitrogen dioxide), hydrocarbons $(\mathrm{HC})$, carbon monoxide $(\mathrm{CO})$, carbon dioxide $\left(\mathrm{CO}_{2}\right)$, and fine particulate matter. Around the world, approximately $50 \%$ of the $\mathrm{NO}_{x}$ emission and $90 \%$ of the $\mathrm{CO}$ emission come from traffic [1]. Consequently, traffic emissions, as an environmentoriented traffic control objective, draw more attention [2-7].

Ecodriving in urban traffic networks using traffic signal information is a significant field of intelligent transportation system (ITS). Technology advancement in wireless communication, specifically Vehicle-to-Infrastructure (V2I) and Vehicle-to-Vehicle (V2V) communications, has the potential of data collection (road, vehicle, and passenger) at any space-time position of traffic networks. Knowledge of traffic signal timings has been proven to benefit the energy use of vehicles by reducing the sudden break and idling time at the intersections $[3,8]$.
Hybrid systems have been an active research topic in the last few years and many results can be found in the control and computer science literature [9]. As a special type of hybrid systems, switched systems have attracted increasing attention $[10,11]$. Wei et al. study the problem of traction control, that is, how to stabilize a wheeled mobile robot (WMR) subject to wheel slippage to a desired configuration [12]. Uthaichana et al. present a hybrid optimal control solution for the power management problem of a parallel hybrid electric vehicle (PHEV), based on a two-mode operation model of PHEV [13].

The green light optimal speed advisory (GLOSA) [14] can synchronize with green signals without stopping at intersections when vehicles are traveling at an optimal speed. In addition, GLOSA helps vehicles keep a driving speed as much as possible to reduce additional acceleration. In [15], authors provided a fast suboptimal solution that suggests an online speed advisory service to the driver for the energy minimization based on GLOSA system. A framework for vehicle's ecodriving was developed in [16] based on the V2I communication technology to enhance vehicle fuel 


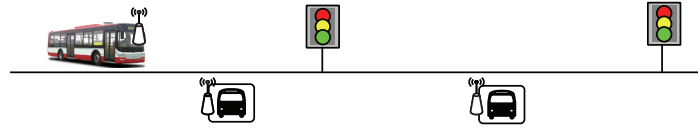

FIGURE 1: V2I communication in intelligent transportation systems.

consumption efficiency. In [17], Asadi and Vahidi propose the use of upcoming traffic signal information within the vehicle's adaptive driving control system to reduce stop time at stop lights and fuel consumption. To achieve this goal an optimization-based control algorithm is formulated that uses short range radar and traffic signal information to schedule an optimum velocity trajectory for the vehicle. More extensive discussion about how to find the energy-optimal velocity for a vehicle using V2I and V2V communication in urban traffic networks can be reviewed in the literature $[18,19]$.

Here we are interested in optimal control of bus transit with minimizing the volume of pollutant of vehicle emissions, such as $\mathrm{NO}_{x}, \mathrm{HC}$, and $\mathrm{CO}_{2}$. The vehicle emission model, vehicle specific power (VSP) model [20, 21], is used as performance index to quantify the vehicle emissions, which is closely related to the vehicle dynamics (speed, acceleration) and the road grade. The referred trajectories induced from the traditional "bus priority" and "green wave" schemes of traffic signal timing are tracked by the driver in order to pass through the intersections without stopping. Using V2I communication technology, not only the information of traffic signal planning but also the estimated total delay at each station is transmitted to drivers for decision making. Physical topology graph is shown in Figure 1. Following from our discussion, we also obtain a suboptimal ecodriving trajectory for bus transit to pass through intersections in green light against the dwell time of bus transit at stations.

This paper is organized as follows: Section 2 modeling the vehicle's hybrid dynamic model with switching laws and guard conditions. The traffic signal timing scheme and the global optimal control model for ecodriving are introduced in Sections 3 and 4, respectively. Section 5 describes the iterative optimization method. In Section 6, we select an approximate sixteen-kilometer traffic route of Beijing bus transit line 1 for simulation to verify the validity of the vehicle's hybrid dynamic model through the numerical solution of the simulation. Conclusions and future works are development in Section 7.

\section{Vehicle's Hybrid Dynamic Model}

The movement of the vehicle is discontinuous, which is interrupted by the passengers regularly getting on and off at the stations by the bus transportation schedule. At the same time, the different vehicle dynamics, such as driving and dwell process, usually lead to the different emission levels of the pollutant. Therefore, it is convenient to build the movement of the vehicle with the hybrid dynamical model. Vehicle's hybrid dynamic model and switching laws among hybrid dynamics are formulated as follows.
Driving Mode. Using the vehicle kinematics, the dynamic of driving mode can be stated as the following equations:

$$
\begin{aligned}
\dot{x}(t) & =v(t), \\
M \dot{v}(t) & =f_{e}(t)-f_{d}(t),
\end{aligned}
$$

where $x(t)$ is the displacement of vehicle, $v(t)$ is the speed of vehicle, $M$ is the vehicle mass, $f_{e}(t)$ is traction force of the engine, and $f_{d}(t)$ means the road forces including aerodynamic drag, rolling resistance, and road grade forces [17].

The road forces $f_{d}(t)$ can be described in detail as

$$
f_{d}(t)=M g \sin \theta+M g C_{r}+\frac{1}{2} \rho_{\alpha}(v(t))^{2} A C_{a},
$$

where $g$ is the gravitational constant, $\theta$ is the road grade angle, $C_{r}$ is the rolling resistance coefficient, $\rho_{\alpha}$ is the mass density of air, $A$ is the cross-sectional area, and $C_{a}$ is the aerodynamic drag coefficient.

When the vehicle dynamics are in the driving mode the vehicle accelerates leaving the station, goes through the intersection with green light, and decelerates into the station. During the driving mode process the vehicle is driving at an expected speed if we neglect the transient acceleration and deceleration process.

Dwell Mode. The location of each station is known, the vehicle dynamics are in the dwell mode when $x(t)=s_{j}$, where $s_{j}$ is the location of station $S n_{j}$. The dynamic of dwell mode can be stated as the following equations:

$$
\begin{aligned}
\dot{x}(t) & =0, \\
M \dot{v}(t) & =0, \\
\dot{\tau}(t) & =1,
\end{aligned}
$$

where $\tau(t)$ is a timer used to record the vehicle's dwell time. When the vehicle leaves the station, the timer is cleared.

The dwell time of vehicle $d_{c}^{j}$ at station $S n_{j}$ includes two parts; one is dwell time of passengers regularly getting on and off $d_{w}^{j}(t)$; the other one is control quantity $\delta_{j}$. $\delta_{j}$ is controlled by the driver, meaning the dwell time after all the passengers have regularly finished getting on and off.

The dwell time of passengers regularly getting on and off $d_{w}^{j}(t)$ can be estimated with the number of waiting passengers $n_{p}^{j}(t)$ at station [23], in which

$$
d_{w}^{j}(t)=g\left(n_{p}^{j}(t)\right)
$$

where $n_{p}^{j}(t)$ is the number of waiting passengers and $g(\cdot)$ is a linear or nonlinear function about the number of passengers.

$\delta_{j}$ is a time independent control variable of vehicle's hybrid dynamic model. The upper bound value of $\delta_{j}$ is $\delta_{\max }^{j}$, so the dwell time of vehicle $d_{c}^{j}$ can be written as the following equation:

$$
d_{c}^{j}=d_{w}^{j}(t)+\delta_{j},
$$

where $d_{c}^{j} \in\left[d_{w}^{j}(t), d_{w}^{j}(t)+\delta_{\max }^{j}\right)$. 


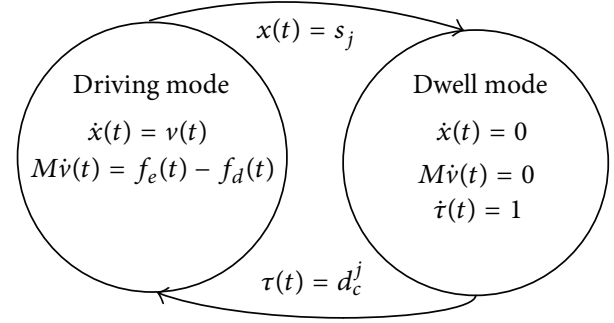

FIGURE 2: Switching laws of vehicle’s hybrid dynamic model.

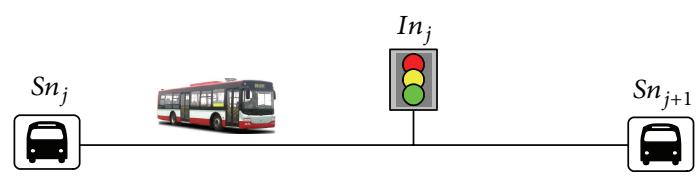

FIGURE 3: Figure of bus transit operation state.

Switching Laws. The vehicle's hybrid dynamic model contains two kinds of switching processes. The one switching process is switching from driving mode to dwell mode; it is autonomous switches; the other switching process is switching from dwell mode to driving mode; it is controlled switches.

Figure 2 describes the switching laws and the guard conditions for the hybrid dynamic model of vehicle. The guard condition from the driving mode to the dwell mode is autonomous as the vehicle arriving stations, while the guard condition from the dwell mode to the driving mode is modeled as control variables of hybrid model such that the timer $\tau(t)$ is equal to the dwell time of vehicle $d_{c}^{j}$.

\section{Traffic Signal Timing}

Suppose there is an intersection between every two adjacent stations. As it is shown in Figure 3, vehicle leaves from any station $S n_{j}$ driving to the downstream adjacent station $S n_{j+1}$ and goes through the intersection $I n_{j}$ with green light. Going through the intersection with green light can reduce vehicle acceleration and deceleration and vehicle brake numbers as well as vehicle delay and emissions. Therefore it is necessary to know the traffic signal timing of intersection in order to ensure the vehicle without stop at intersection.

In our research, the traffic signal timing at intersections including cycle, split, and offset times is deterministic and given. Under the assumption that the information of signal timing of the traffic lights can be shared with the vehicle via the V2I communication, it is possible to formulate mathematically the state evolution of the traffic lights as follows:

$$
L_{j}= \begin{cases}1, & r T+t_{g}^{j} \leq t_{I}^{j}<t_{g}^{j}+t_{g}+r T, \\ 0, & t_{g}^{j}+t_{g}+r T \leq t_{I}^{j}<t_{g}^{j}+(r+1) T,\end{cases}
$$

where $L_{j}$ is the state of traffic light (red or green) at intersection $I n_{j}, t_{I}^{j}$ is the time instant at which the vehicle arrives at the intersection $I n_{j}, T$ is the cycle, $t_{g}^{j}$ and $t_{g}$ are the start instant and duration of the green phase, respectively, and $r=1,2, \ldots$ is the number of cycles.

The offset of the neighboring traffic lights is set as

$$
t_{o}^{j}=t_{g}^{j+1}-t_{g}^{j}=\frac{i_{j+1}-i_{j}}{\bar{v}}
$$

where $\bar{v}$ is the "green wave" speed, $i_{j}$ is the position of intersection $I n_{j}$, and $i_{j+1}$ is the position of upstream intersection $I n_{j+1}$. The "green wave" timing scheme of adjacent traffic lights makes the vehicle pass through the next intersection without stop, which has been extensively studied in the field of traffic engineering to reduce delay.

We restrict the vehicle to pass through the intersections in the driving mode of hybrid model without stop, for intersection $I n_{j}$, satisfying

$$
\left(x_{j}\left(t_{I}^{j}\right)=i_{j}\right) \wedge\left(L_{j}=1\right),
$$

where " $\wedge$ " is on behalf of the logic operation "and."

\section{Global Optimal Problem}

Objective Function. Take the minimum of emissions $\left(\mathrm{NO}_{x}\right.$, $\mathrm{HC}, \mathrm{CO}$, and $\mathrm{CO}_{2}$ ) as the target. The vehicle specific power (VSP) model [20] is used as the performance index to measure the pollutants of vehicle emissions. VSP is a proxy variable for engine load and is defined as the source power per unit mass. The calculation formula of the VSP is as follows:

$$
\begin{aligned}
\mathrm{VSP}= & v\left[a \times\left(1+\xi_{i}\right)+g \times \theta+g \times C_{r}\right] \\
& +\frac{1}{2} \rho_{\alpha} \frac{C_{a} \times A}{M}\left(v+v_{m}\right)^{2} \times v,
\end{aligned}
$$

where $v$ is vehicle speed, $a$ is vehicle acceleration, $\xi_{i}$ is vehicle mass factor, $g$ is acceleration of gravity, $\theta$ is vertical rise/slope length, $C_{r}$ is coefficient of rolling resistance, $\rho_{\alpha}$ is ambient air density, $C_{a}$ is drag coefficient, $A$ is frontal area of the vehicle, $M$ is vehicle mass, and $v_{m}$ is wind speed.

Actually the calculated value of VSP through (9) is dimensionless and has not any actual physical meaning. Table 1 is the VSP modes and the average modal emission rates of each. By querying Table 1, we can obtain the emissions. Emissions can be expressed as

$$
E=f(\mathrm{VSP}),
$$

where $E$ is the emissions and $f(\cdot)$ is a piecewise function.

Constraint Conditions. Ensuring that the travel time vehicle must arrive at the terminal station $S n_{N}$ at the moment $t_{f}, t_{S}^{N}=$ $t_{f}$. The speed $v_{j}(t)$, acceleration $a_{j}(t)$, and traction force of the engine $f_{e}^{j}(t)$ values must be in the threshold range, $v_{\min } \leq$ $v_{j}(t) \leq v_{\max }, a_{\min } \leq a_{j}(t) \leq a_{\max }$, and $f_{e}^{\min } \leq f_{e}^{j}(t) \leq f_{e}^{\max }$.

Global Optimal Model. Consider the vehicle's hybrid dynamic model of vehicle, the real-time traffic information of the signal planning of intersections, and residence time of stations. Minimizing the emission index of the whole route in the 
TABLE 1: VSP modes and the average modal emission rates of each [22].

\begin{tabular}{|c|c|c|c|c|c|}
\hline VSP & VSP mode & $\mathrm{CO}_{2}(\mathrm{~g} / \mathrm{s})$ & $\mathrm{CO}(\mathrm{g} / \mathrm{s})$ & $\mathrm{NO}_{x}(\mathrm{~g} / \mathrm{s})$ & $\mathrm{HC}(\mathrm{g} / \mathrm{s})$ \\
\hline VSP $<-2$ & 1 & 1.54369 & 0.01103 & 0.00101 & 0.00090 \\
\hline$-2 \leq \mathrm{VSP}<0$ & 2 & 1.60441 & 0.00872 & 0.00104 & 0.00090 \\
\hline $0 \leq \mathrm{VSP}<1$ & 3 & 1.13083 & 0.00468 & 0.00042 & 0.00084 \\
\hline $1 \leq \mathrm{VSP}<4$ & 4 & 2.38626 & 0.01215 & 0.00161 & 0.00103 \\
\hline $4 \leq \mathrm{VSP}<7$ & 5 & 3.21025 & 0.01673 & 0.00264 & 0.00125 \\
\hline $7 \leq \mathrm{VSP}<10$ & 6 & 3.95773 & 0.02327 & 0.00379 & 0.00166 \\
\hline $10 \leq \mathrm{VSP}<13$ & 7 & 4.75201 & 0.02932 & 0.00510 & 0.00209 \\
\hline $13 \leq \mathrm{VSP}<16$ & 8 & 5.37422 & 0.03694 & 0.00637 & 0.00233 \\
\hline $16 \leq \mathrm{VSP}<19$ & 9 & 5.94005 & 0.04951 & 0.00766 & 0.00282 \\
\hline $19 \leq \mathrm{VSP}<23$ & 10 & 6.42751 & 0.06376 & 0.00991 & 0.00299 \\
\hline $23 \leq \mathrm{VSP}<28$ & 11 & 7.06599 & 0.10538 & 0.01269 & 0.00379 \\
\hline $28 \leq \mathrm{VSP}<33$ & 12 & 7.61770 & 0.24781 & 0.01438 & 0.00457 \\
\hline $33 \leq \mathrm{VSP}<39$ & 13 & 8.32244 & 0.41307 & 0.01597 & 0.00570 \\
\hline $39 \leq \mathrm{VSP}$ & 14 & 8.47503 & 0.62466 & 0.01672 & 0.00716 \\
\hline
\end{tabular}

scheduled transportation interval $\left[0, t_{f}\right]$, we can obtain the global optimal control model for ecodriving as follows:

$$
\begin{aligned}
\operatorname{minimize} & J_{\text {total }}=\int_{t=0}^{t=t_{f}} f(\operatorname{VSP}(v(t), a(t))) d t \\
\text { s.t. } & \text { vehicle dynamics }(1),(2),(3) \\
& \text { residence times }(4),(5)
\end{aligned}
$$$$
\text { signal timing }(6),(7),(8) \text {, }
$$

where $t_{S}^{N}=t_{f}$ is the time to arrive at station $S n_{N}$. The constraint condition (8) for the "green wave" of the signal timing represents a set of disjointed time intervals at the same intersection. Therefore, the constraint conditions are nonconvex sets and the solution of the optimal ecodriving of vehicle has to be considered as a suboptimal way.

\section{Iterative Optimization Method}

Dwell time of passengers getting on and off is a disturbance to the global optimal control. It is difficult to predict the values of $d_{w}^{j}(t)$ in advance for each station. In order to solve this problem we should update the dwell time $d_{w}^{j}(t)$ through V2I communication at the moment when the vehicle arrives at each station.

Suppose there are $N$ stations and $N-1$ intersections. The space discretization is carried out according to the position of the station. All optimal solutions can be solved in the iterative optimization; for each step, the vehicle reaches to the next downstream station. Every step there are $N_{p}$ stations and $N_{c}$ inputs. $N_{p}$ means how "far" we wish the future to be predicted for, where $N_{p} \leq N-1$ is positive integer. $N_{c}$ dictates the number of parameters used to capture the future control trajectory, $N_{c} \leq N_{p}$. There are $N-N_{p}+1$ iterative optimization steps. $F_{e}^{k}$ and $D_{c}^{k}$ are input vectors,
$F_{e}^{k}=\left[f_{e}^{1}(t), \ldots, f_{e}^{j}(t), \ldots, f_{e}^{N_{c}}(t)\right], D_{c}^{k}=\left[d_{c}^{1}, \ldots, d_{c}^{j}, \ldots\right.$, $\left.d_{c}^{N_{c}}\right]$. For $k$ th step $k=1,2, \ldots, N-N_{p}+1$, we have

$$
J_{t}(k)=\min _{D_{c}^{k}, F_{e}^{k}} \sum_{j=k}^{k-1+N_{p}}\left(J_{d r}^{j}+J_{d w}^{j}\right),
$$

where $J_{t}(k)$ is the total emission of $k$ th step iterative optimization and $J_{d r}^{j}$ and $J_{d w}^{j}$ are the emissions during the driving mode and dwell mode, respectively.

The performance indexes $J_{d r}^{j}$ and $J_{d w}^{j}$ of the vehicle's hybrid dynamics can be described as the following equations, respectively, with more details, for $j=1,2, \ldots, N-1$ :

$$
\begin{gathered}
J_{d r}^{j}=\int_{t_{S}^{j}+d_{c}^{j}}^{t_{S}^{j+1}} f\left(\operatorname{VSP}\left(a_{j}(t), v_{j}(t)\right)\right) d t \\
J_{d w}^{j}=\int_{t_{S}^{j}}^{t_{S}^{j}+d_{c}^{j}} f\left(\operatorname{VSP}\left(a_{j}(t), v_{j}(t)\right)\right) d t,
\end{gathered}
$$

where $t_{S}^{j}$ and $t_{S}^{j+1}$ are the time instant at which the vehicle arrives at the stations $S n_{j}, S n_{j+1}$, respectively.

For $k$ th step the dimension of each input vector is $N_{c}$. We only implement the first element of these input vectors, that is, $f_{e}^{1}(t)$ and $d_{c}^{1}$, while ignoring the rest of the elements (except the last step). When the next step arrives, the more recent measurement is taken from the state vector for calculation of the new sequence of input vector. This procedure is repeated in real time during the process of iterative optimization. The optimal emission for $k$ th step is $J^{*}(k), J^{*}(k)=\left(J_{d r}^{k}+J_{s t}^{k}\right)$.

Notice that when the vehicle arrives at station $S n_{N-N_{p}+1}$, that is, the last step, the number of stations at the downstream remains less than $N_{p}$. Then all elements of $F_{e}^{N-N_{p}+1}$ and $D_{c}^{N-N_{p}+1}$ are effective. The optimal emission of last step is $J^{*}\left(N-N_{p}+1\right), J^{*}\left(N-N_{p}+1\right)=J_{t}\left(N-N_{p}+1\right)$. 


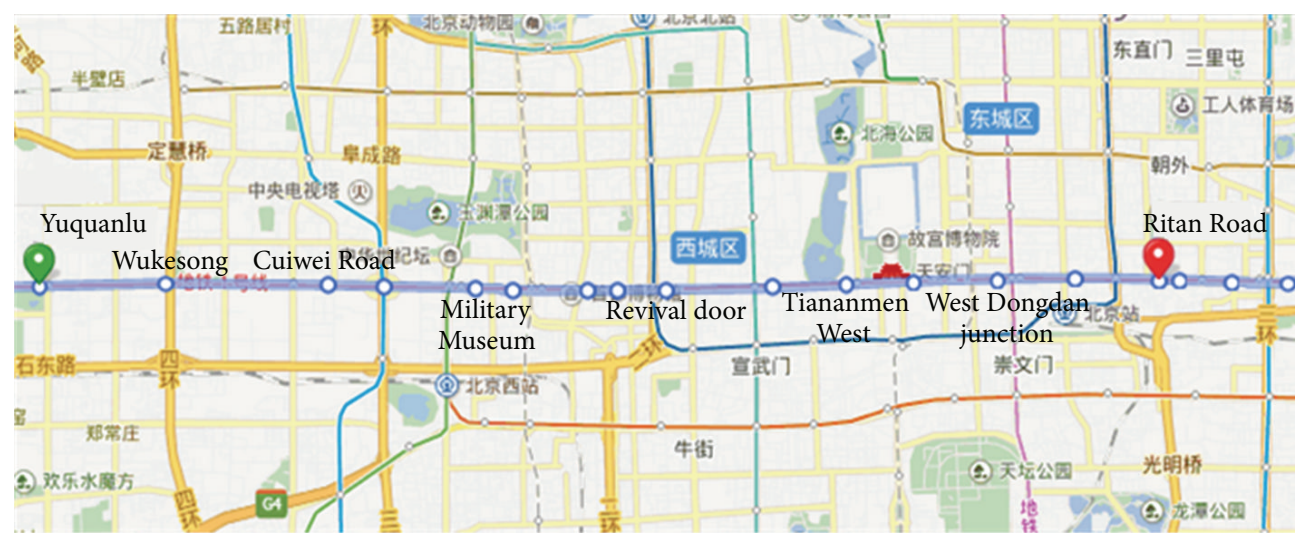

FIGURE 4: Bus route of bus transit line one in Beijing city.

TABLE 2: Vehicle performance parameter.

\begin{tabular}{lccccc}
\hline Para. & $M(\mathrm{~kg})$ & $P(\mathrm{kw})$ & $f_{e}^{\min }(\mathrm{N})$ & $f_{e}^{\max }(\mathrm{N})$ & Physical dimension \\
\hline Values & $17000(28000)$ & 213 & 6000 & 98000 & $17980 * 2550 * 3100$ \\
\hline
\end{tabular}

The total optimal emissions $J_{\text {total }}^{*}, J_{\text {total }}^{*}=\sum_{k=1}^{N-N_{p}} J^{*}(k)+$ $J_{t}\left(N-N_{p}+1\right)$.

In the above iterative optimization method process, each step of the iterative optimization is an optimization problem. Main steps are including pruning algorithm, searching feasible path, and optimizing. More detailed steps are as follows.

Step 1. Determine the initial range of state variables.

Step 2. By pruning algorithm calculates feasible regions of each state variable.

Step 3. Determine feasible paths and calculate feasible regions of state variables for each path.

Step 4. Calculate feasible path's optimal solution through nonlinear programming.

Step 5. Repeat Step 4, until every feasible path has been calculated.

Step 6. Search for the optimal feasible path, that is, feasible path corresponding to the minimum value of the optimal index.

Remark 1. The classical model predictive control is a receding horizon optimization process. In our optimization method, the dynamic iterative process is receding along the spatial positions of the stations.

\section{Experiments}

A segment of bus transportation route of bus transit line 1 in Beijing is chosen as the simulation scene. This transportation route consists of fifteen stations, from the Yuquan Road station to the Ritan Road station, respectively, as seen in
Figure 4, and fourteen traffic intersections with the twophase signal timing scheme. The spatial displacement of the stations and the traffic intersections are obtained from the Baidu Digital Map.

Bus transit line one is BJ6180C8CTD model of 18-meter LNG (liquefied natural gas) articulated vehicle made by FOTON motor incorporated company. The vehicle performance parameters are shown in Table 2. The unladen mass of vehicle is 17000 kilograms and the total mass is 28000 kilograms. Rated power $P$ of the vehicle is 213 kilowatts. The physical dimension of the vehicle is 17.98-meter long, 2.55meter wide, and 3.1-meter high. See Table 2 for details.

Parameter values for calculating vehicle drag are shown in Table 3. The slope of the road is zero. The cross section of vehicle $A$ is calculated by the height of the vehicle times the width of the vehicle.

The other model and simulation parameters, such as the station position, intersection position, speed limit of the vehicle, acceleration limit of vehicle, simulation times, and total number of stations are listed in Table 4.

Table 5 lists the parameters of traffic signal timing of the fourteen intersections. The cycle time is 120 seconds; the green time of the vehicle passing phase is 30 seconds. The offset of the neighboring intersections is 113-31-2090-99-26-10-72-74-12-102-113-8 seconds, respectively. In this simulation, the start time $t_{g}^{1}$ of the green phase of the first intersection is 0 .

Simulation results of the total emissions $J_{\text {total }}^{*}$ are 2420 grams. The total delay time at each station $d_{c}$, the maximum traction force $f_{e}^{m}$ (i.e., the maximum value of each stage in Figure 7), the optimal speeds $v^{*}$, the optimal acceleration $a^{*}$, the optimal arriving time of each station $t_{S}^{j^{*}}$, and the optimal arriving time of each intersection $t_{I}^{j^{*}}$ are shown in Table 6.

Figure 5 gives the spatiotemporal diagram of optimal driving route for the public transits line one. Vehicle delays at 
TABLE 3: Road forces calculation parameters.

\begin{tabular}{lcccccc}
\hline Para. & $g\left(\mathrm{~m} / \mathrm{s}^{2}\right)$ & $\theta\left(^{\circ}\right)$ & $C_{r}$ & $C_{a}$ & $\rho_{\alpha}\left(\mathrm{kg} / \mathrm{m}^{3}\right)$ & $A\left(\mathrm{~m}^{2}\right)$ \\
\hline Values & 9.81 & 2 & 0.015 & 0.55 & 1.207 & 7.91 \\
\hline
\end{tabular}

TABLE 4: Model and simulation parameters.

\begin{tabular}{|c|c|c|c|c|c|c|c|c|}
\hline Para. & $S(\mathrm{~m})$ & $I(\mathrm{~m})$ & $a_{j}(t)\left(\mathrm{m} / \mathrm{s}^{2}\right)$ & $v_{j}(t)(\mathrm{m} / \mathrm{s})$ & $t_{f}(\mathrm{~s})$ & $N_{c}$ & $N_{p}$ & $N$ \\
\hline Values & $\begin{array}{c}0 \\
1800 \\
4200 \\
5000 \\
6300 \\
6900 \\
8000 \\
8400 \\
9100 \\
10700 \\
11800 \\
12800 \\
14000 \\
15100 \\
16300\end{array}$ & $\begin{array}{c}1100 \\
2900 \\
4700 \\
5900 \\
6600 \\
7200 \\
8300 \\
9000 \\
9500 \\
11200 \\
12300 \\
12900 \\
14900 \\
16200\end{array}$ & {$[0,3.5]$} & {$[0,14]$} & 2100 & 4 & 4 & 15 \\
\hline
\end{tabular}

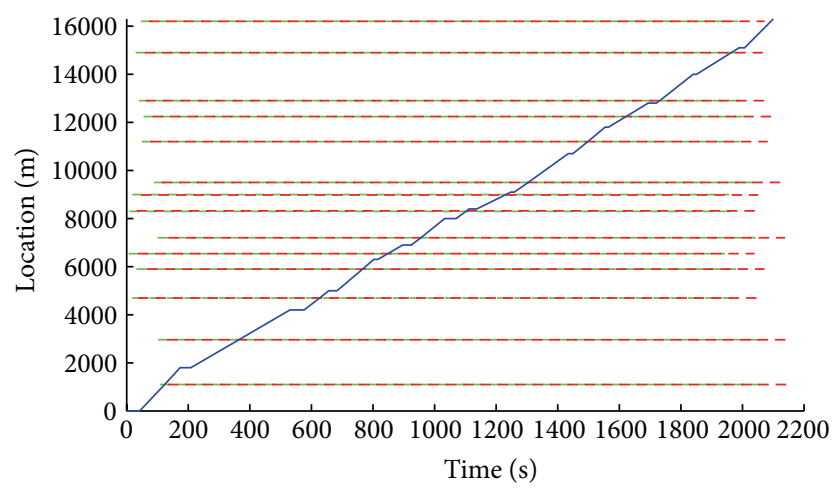

FIGURE 5: Spatiotemporal figure of driving routes.

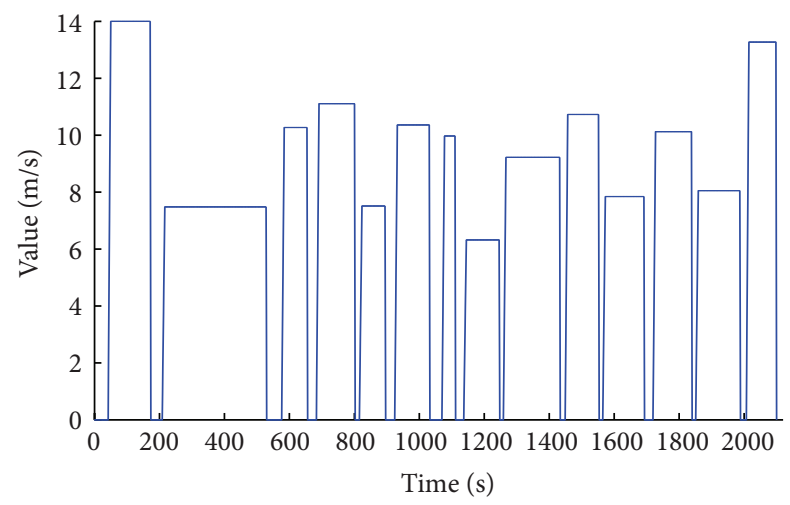

Figure 6: Speed suggestions.

each station are different. The vehicle crosses the intersections without stop with optimal speed $v^{*}$.

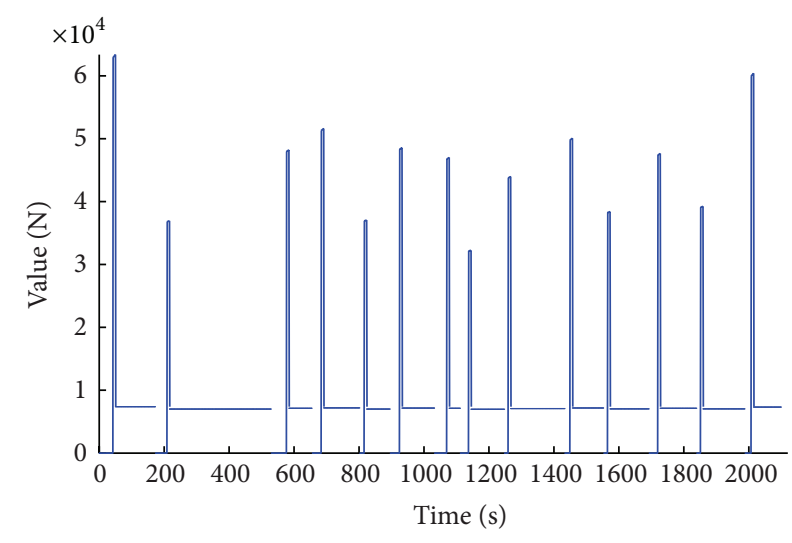

Figure 7: Traction force of engine.

Figure 6 shows the variation curve of the optimal vehicle speed suggestion with time. The curve is periodic. In each period, the driving speeds are different. This is caused by the actual position of the stations and the total delay times.

Vehicle traction force of engine variation curves with time is shown in Figure 7. It is periodic too. In each period, the traction force of engine is zero at the station; it surges at the acceleration process and at last decreases to a constant value and maintains stability at driving mode.

\section{Conclusions}

According to the actual operation of the bus transit, vehicle's hybrid dynamic model is established. The dwell time of the vehicle at each station which includes two parts is proposed. Previous works have proved how the V2I has a positive impact on the energy consumption. In this work, further improvement of ecodriving to lower vehicle emission 
Table 5: Traffic light timing.

\begin{tabular}{lcccc}
\hline Para. & $T(\mathrm{~s})$ & $t_{g}(\mathrm{~s})$ & $t_{g}^{1}(\mathrm{~s})$ & $t_{0}^{j}(\mathrm{~s})$ \\
\hline Values & 120 & 30 & 0 & $113-31-20-90-99-26-10-72-74-12-102-113-8$ \\
\hline
\end{tabular}

TABLE 6: Simulation results and emission index.

\begin{tabular}{lc}
\hline Variables & Values \\
\hline$d_{c}(\mathrm{~s})$ & $42-35-46-27-13-28-37-25-11-15-11-26-11-18$ \\
$f_{e}^{m}(\mathrm{~N})$ & $63380-36940-48227-51616-37069-48577-47011-32249-43976-50076-38406-47647-39251-60405$ \\
$t_{I}^{j^{*}}(\mathrm{~s})$ & $123-356-627-766-856-955-1102-1232-1304-1498-1630-1731-1964-2092$ \\
$t_{S}^{j^{*}}(\mathrm{~s})$ & $0-173-530-656-803-896-1033-1112-1248-1434-1554-1693-1840-1988-2100$ \\
$a^{*}\left(\mathrm{~m} / \mathrm{s}^{2}\right)$ & $2.8-1.5-2-2.2-1.5-2-2-1.3-1.8-2.1-1.6-2-1.6-2.7$ \\
$v^{*}(\mathrm{~m} / \mathrm{s})$ & $14-7.5-10-11-7.5-10.4-10-6.3-9.2-10.7-7.8-10-8-13$ \\
$J_{\text {total }}^{*}(\mathrm{~g})$ & 2420 \\
\hline
\end{tabular}

considering dwell time of vehicle is presented. Global optimal control for minimizing the VSP index is solved based on the vehicle's hybrid dynamic model with the iterative optimization method. The simulation results show that vehicle can pass through intersections without stopping under the different scenes of waiting passenger. Further works will consider multiobjective optimization and bus transit embedded in traffic flow.

\section{Conflict of Interests}

The authors declare that there is no conflict of interests regarding the publication of this paper.

\section{Acknowledgment}

This work was supported by the National Science Foundation of China (NSFC, Grant no. 61374076).

\section{References}

[1] A. Nagurney, "Congested urban transportation networks and emission paradoxes," Transportation Research Part D: Transport and Environment, vol. 5, no. 2, pp. 145-151, 2000.

[2] M. A. S. Kamal, M. Mukai, J. Murata, and T. Kawabe, "Ecological vehicle control on roads with up-down slopes," IEEE Transactions on Intelligent Transportation Systems, vol. 12, no. 3, pp. 783794, 2011.

[3] M. Ferreira and P. M. D'Orey, "On the impact of virtual traffic lights on carbon emissions mitigation," IEEE Transactions on Intelligent Transportation Systems, vol. 13, no. 1, pp. 284-295, 2012.

[4] C. Li and S. Shimamoto, "Dynamic traffic light control scheme for reducing $\mathrm{CO}_{2}$ emissions employing ETC technology," International Journal of Managing Public Sector Information and Communication Technologies, vol. 2, no. 1, pp. 1-12, 2011.

[5] A. Stevanovic, J. Stevanovic, K. Zhang, and S. Batterman, "Optimizing traffic control to reduce fuel consumption and vehicular emissions," Transportation Research Record, no. 2128, pp. 105113, 2009.

[6] S. K. Zegeye, B. De Schutter, J. Hellendoorn, E. A. Breunesse, and A. Hegyi, "Integrated macroscopic traffic flow, emission, and fuel consumption model for control purposes," Transportation Research Part C: Emerging Technologies, vol. 31, pp. 158-171, 2013.

[7] S. Zegeye, B. De Schutter, H. Hellendoorn, and E. Breunesse, "Modelbased traffic control for balanced reduction of fuel consumption, emissions, and travel time," in Proceedings of the 12th IFAC Symposium on Transportation Systems, pp. 149-154, Redondo Beach, Calif, USA, September 2009.

[8] M. Ferreira, R. Fernandes, H. Conceição, W. Viriyasitavat, and O. K. Tonguz, "Self-organized traffic control," in Proceedings of the 7th ACM International Workshop on VehiculAr InterNETworking (VANET '10), pp. 85-89, Chicago, Ill, USA, September 2010.

[9] A. J. ven der Schaft and H. Schumacher, An Introduction to Hybrid Dynamical Systems, vol. 251 of Lecture Notes in Control and Information Sciences, Springer, London, UK, 2000.

[10] T. I. Seidman, "Optimal control for switching systems," in Proceedings of the 21st Annual Conference on Information Sciences and Systems (CISS '78), pp. 485-489, Baltimore, Md, USA, 1978.

[11] J. Yong, "Systems governed by ordinary differential equations with continuous, switching and impulse controls," Applied Mathematics and Optimization, vol. 20, no. 3, pp. 223-235, 1989.

[12] S. Wei, K. Uthaichana, M. Žefran, and R. DeCarlo, "Hybrid model predictive control for the stabilization of wheeled mobile robots subject to wheel slippage," IEEE Transactions on Control Systems Technology, vol. 21, no. 6, pp. 2181-2193, 2013.

[13] K. Uthaichana, S. Bengea, R. DeCarlo, S. Pekarek, and M. Zefran, "Hybrid model predictive control tracking of a sawtooth driving profile for an HEV," in Proceedings of the American Control Conference (ACC '08), pp. 967-974, IEEE, Seattle, Wash, USA, June 2008.

[14] K. Katsaros, R. Kernchen, M. Dianati, and D. Rieck, "Performance study of a Green Light Optimized Speed Advisory (GLOSA) application using an integrated cooperative ITS simulation platform," in Proceedings of the 7th International Wireless Communications and Mobile Computing Conference (IWCMC '11), pp. 918-923, Istanbul, Turkey, July 2011.

[15] G. De Nunzio, C. Canudas de Wit, P. Moulin, and D. Di Domenico, "Eco-driving in urban traffic networks using traffic signal information," in Proceedings of the 52nd IEEE Conference on Decision and Control (CDC '13), pp. 892-898, Firenze, Italy, December 2013. 
[16] H. Rakha and R. K. Kamalanathsharma, "Eco-driving at signalized intersections using V2I communication," in Proceedings of the 14th IEEE International Intelligent Transportation Systems Conference (ITSC '11), pp. 341-346, IEEE, Washington, DC, USA, October 2011.

[17] B. Asadi and A. Vahidi, "Predictive cruise control: utilizing upcoming traffic signal information for improving fuel economy and reducing trip time," IEEE Transactions on Control Systems Technology, vol. 19, no. 3, pp. 707-714, 2011.

[18] X. Ma, "Towards intelligent fleet management: local optimal speeds for fuel and emissions," in Proceedings of the 16th International IEEE Conference on Intelligent Transportation Systems: Intelligent Transportation Systems for All Modes (ITSC '13), pp. 2201-2206, The Hague, The Netherlands, October 2013.

[19] H. Noori and M. Valkama, "Impact of VANET-based V2X communication using IEEE 802.11p on reducing vehicles traveling time in realistic large scale urban area," in Proceedings of the 2nd IEEE International Conference on Connected Vehicles and Expo (ICCVE '13), pp. 654-661, Las Vegas, Nev, USA, December 2013.

[20] J. Jimenez-Palacios, Understanding and quantifying motor vehicle emissions with vehicle specific power and TILDAS remote sensing [Ph.D. thesis], Massachusetts Institute of Technology, Cambridge, Mass, USA, 1999.

[21] Y. Hao, L. Yu, G. Song et al., "Analysis of driving behavior and emission characteristics for diesel transit buses using PEMS measurements," in Proceedings of the 89th Transportation Research Board Annual Meeting, Washington, DC, USA, January 2009.

[22] H. Frey, A. Unal, J. Chen, S. Li, and C. Xuan, "Methodology for developing modal emission rates for EPA's multi-scale motor vehicle \& equipment emission system," Tech. Rep. EPA420-R02-027, Environmental Protection Agency, Washington, DC, USA, 2002.

[23] T. Wang, R. Zhang, L. Zhang et al., "Analysis of the bus rapid transit (BRT) platform stop delay and the boarding time," in Proceedings of the 5th Chinese TongZhou Transport Forum, Shanghai, China, 2008. 


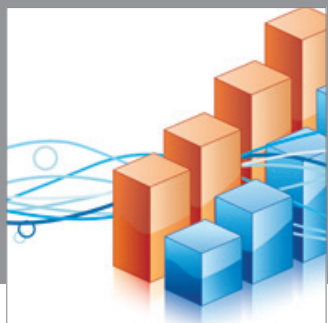

Advances in

Operations Research

mansans

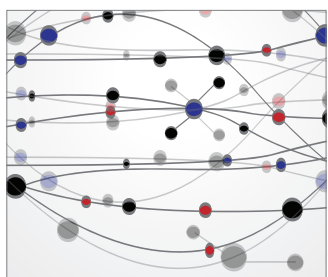

The Scientific World Journal
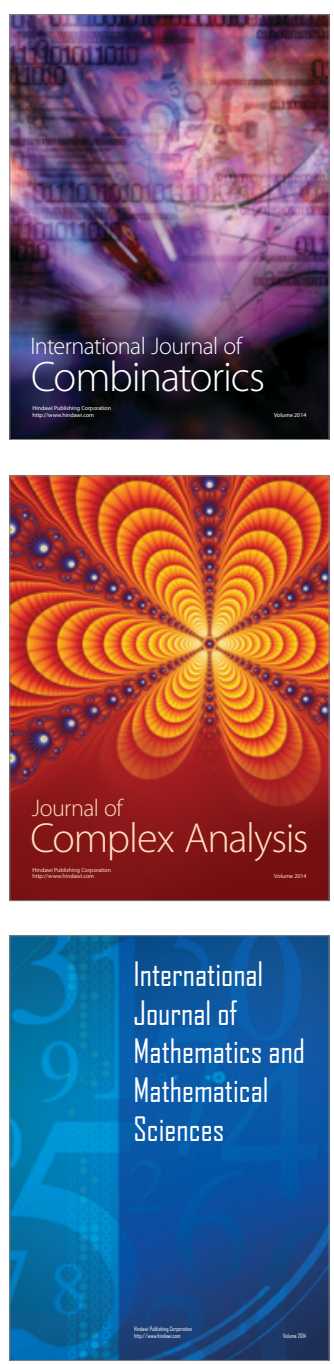
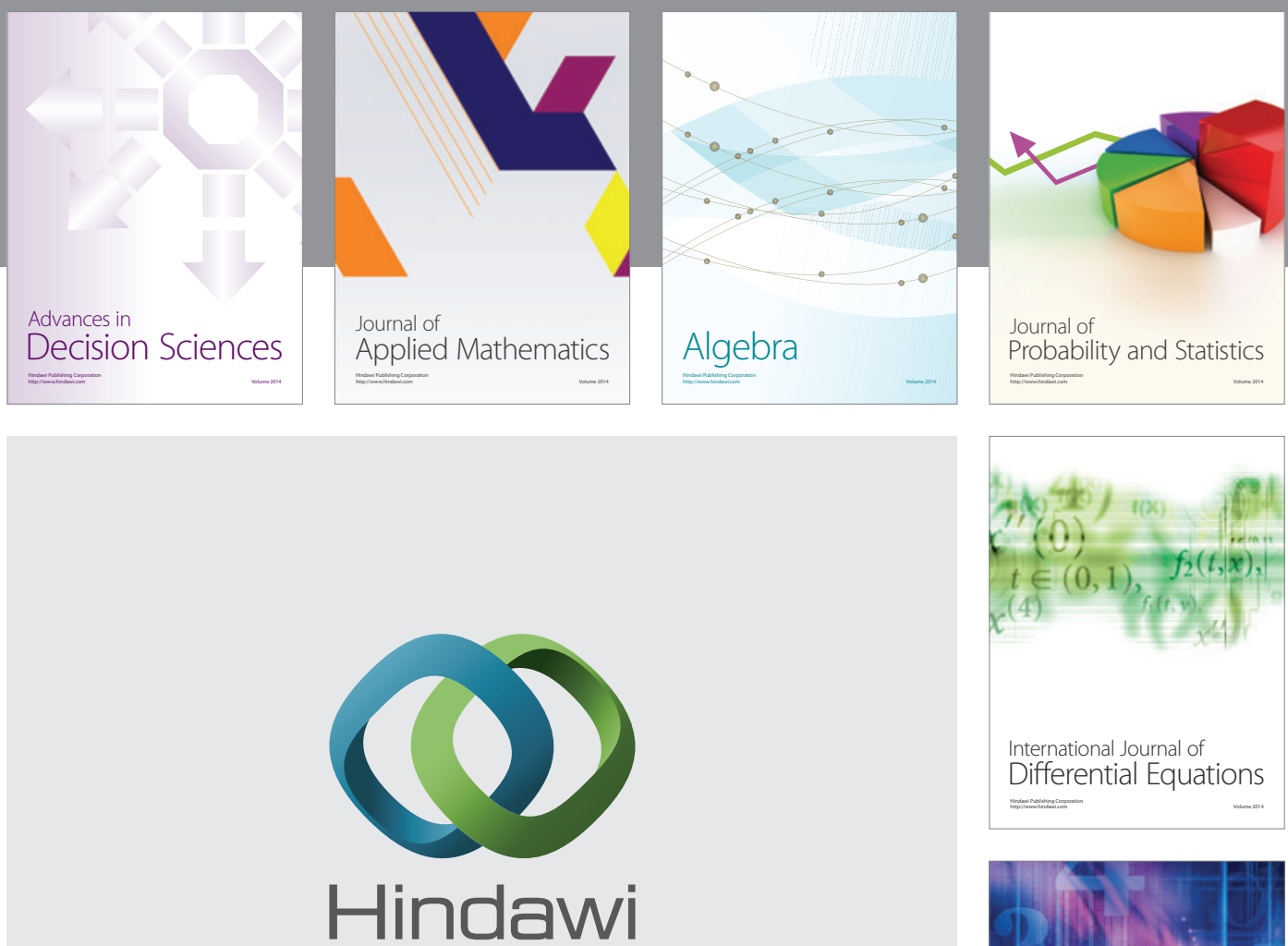

Submit your manuscripts at http://www.hindawi.com
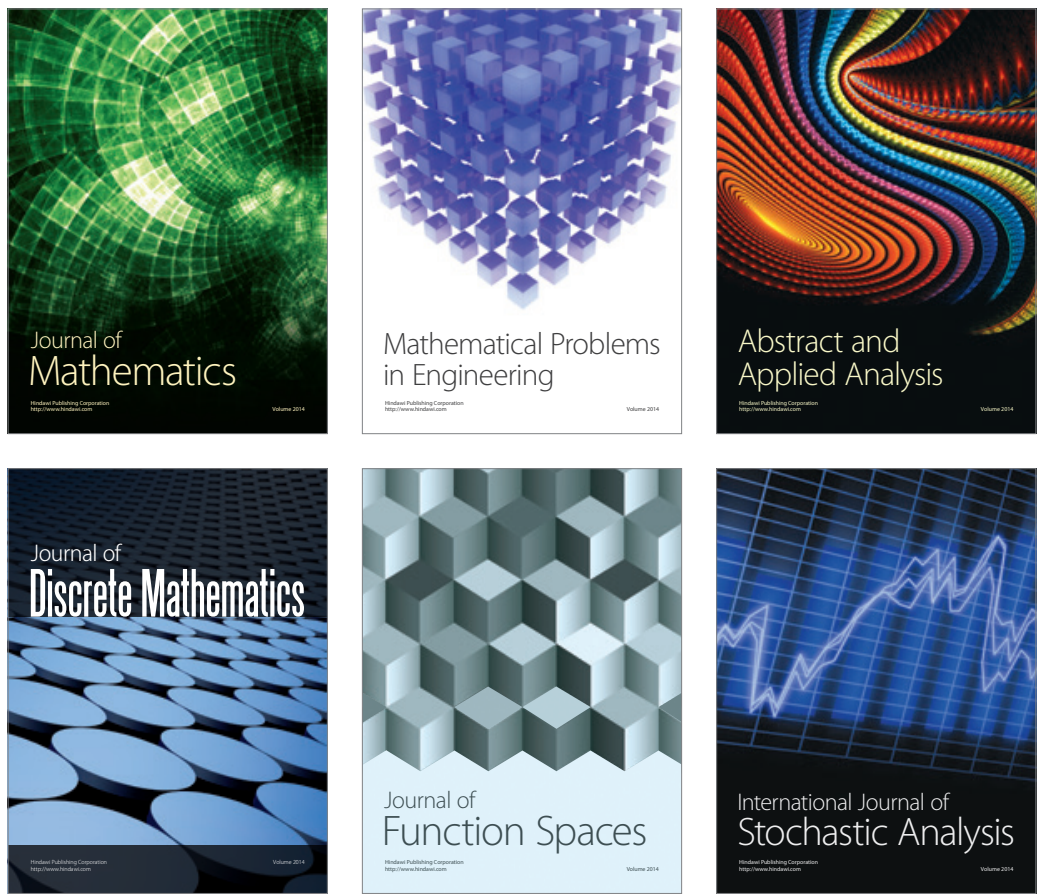

Journal of

Function Spaces

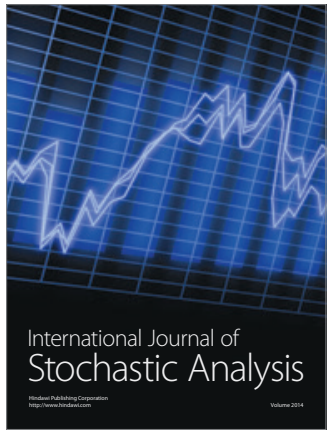

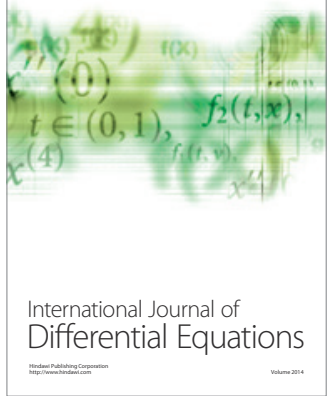
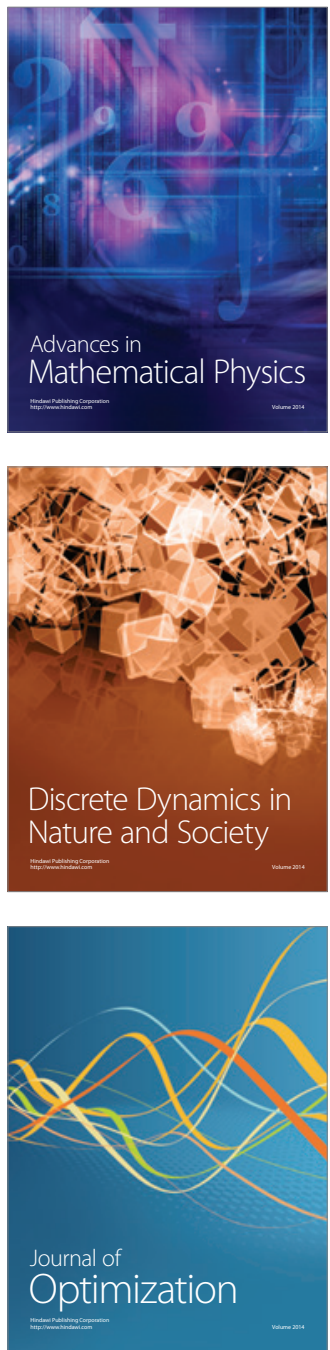